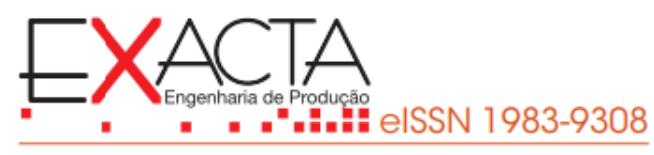

\title{
DESMISTIFICANDO A EQUAÇÃO DE NIOSH PARA TAREFAS COMPOSTAS: UM ROTEIRO DE APLICAÇÃO
}

\section{DEMYSTIFYING THE NIOSH EQUATION FOR MULTI-TASKS: A PRACTICAL ROADMAP APPLICATION}

Recebido em: 02 ago. 2020

Aprovado em: 26 jan. 2021

Versão do autor aceita publicada online: 26 jan. 2021

Publicado online: 12 maio 2021

Como citar esse artigo - American Psychological Association (APA):

Moura, H. M., Franz, Luis A. S., \& Andrade, I. F. (2022, out./dez.). Desmistificando a equação de NIOSH para tarefas compostas: Um roteiro de aplicação. Exacta. 20(4), 948-968. https://doi.org/10.5585/exactaep.2022.17832.

Submeta seu artigo para este periódico $\beta$

Dados Crossmark 


\title{
Desmistificando a equação de NIOSH para tarefas compostas: Um roteiro de aplicação
}

\author{
Demystifying the NIOSH equation for multi-tasks: A practical roadmap \\ application
}

Henrique Martim de Moura ${ }^{1}$

Luis Antonio Santos Franz ${ }^{2}$

Isabela Fernandes Andrade ${ }^{3}$

${ }^{1}$ Engenheiro de Produção

Universidade Federal de Pelotas - UFPel.

Pelotas, Rio Grande do Sul - Brasil.

henriquemdemoura@gmail.com

2 Doutor em Engenharia de Produção

Universidade Federal do Rio Grande do Sul - UFRGS.

Porto Alegre, Rio Grande do Sul - Brasil.

luisfranz@gmail.com

${ }^{3}$ Doutora em Arquitetura e Urbanismo Universidade Federal de Santa Catarina - UFSC. Florianópolis, Santa Catarina - Brasil. acessiarq@gmail.com
Resumo: A Equação de NIOSH é difundida e utilizada no Brasil por profissionais e acadêmicos. Porém, um dos principais problemas é a aplicação da Equação de NIOSH para tarefas simples, em contextos de tarefas que são caracterizadas como tarefas compostas. Não obstante, há uma lacuna importante nestes trabalhos, no que compete à demonstração de forma mais didática do seu uso. Sendo assim, o presente trabalho caracteriza-se como um estudo de caso, onde a equação de NIOSH foi aplicada seguindo cinco passos metodológicos em uma tarefa composta comum no contexto de macromercados e supermercados. Concluímos que a utilização da Equação de NIOSH composta, desde que feita com parcimônia e utilizando-se os valores corretos das frequências durante os procedimentos de cálculo, pode trazer uma avaliação mais acurada dos riscos relativos à manutenção de cargas em atividades complexas; evitando percepções equivocadas das situações de trabalho e consequentemente em danos à saúde do trabalhador.

Palavras-chave: Equação de NIOSH composta. Manutenção de cargas. Avaliação ergonômica.

Abstract: The NIOSH equation is disseminated and used in Brazil by professionals and academics. However, one of the main problems is applying the NIOSH Equation for simple task contexts characterized as multi-tasks. Nevertheless, there is an important gap in these works in demonstrating the most didactic use of them. Thus, the present work is described as a case study. The NIOSH equation was applied following five methodological steps in a multi-task common in the context of macro markets and supermarkets. We conclude that using NIOSH Equation for multi-tasks provided that it is done sparingly and using the correct values of frequencies during the calculation procedures. Can bring a more accurate assessment of the risks related to the maintenance of loads in complex activities, avoiding misperceptions of work situations and, consequently, damage to workers' health.

Keywords: NIOSH Equation for multi-task. Manual handling loads. Ergonomic evaluation. 


\section{Indrodução}

Compreender o processo de movimentação manual de cargas e suas consequências para a saúde dos trabalhadores consiste ainda em um desafio presente no cotidiano do trabalho, incluindo-se aqui a realidade brasileira. De certa maneira, empresas no Brasil experimentam um paradoxo, enquanto de um lado organizações debatem a implementação de conceitos como inteligência artificial, internet das coisas, inteligência de dados dentro outros, que juntos formam a chamada Indústria 4.0, de outro lado temos empresas com grandes dificuldades em buscar soluções para desafios básicos, como acidentes e afastamentos ligados a movimentação manual de cargas.

Desde a década de 1990, a Ergonomia vem projetando-se no Brasil, principalmente após a publicação da Norma Regulamentadora № 17 (BRASIL, 2007), que versa sobre o tema. A consolidação da Associação Brasileira de Ergonomia (ABERGO), que foi criada em 1983, também foi essencial para disseminação desta área no país, estimulando também a ênfase nesta disciplina em cursos de pósgraduação. Mesmo com a ampliação da Ergonomia no Brasil, muitos acidentes e adoecimentos relacionados à movimentação manual de cargas ainda ocorrem, sendo que, lida e Guimarães (2016) relatam que a movimentação de cargas, é a atividade que mais afasta e causa transtornos por lesões musculares, tendo uma taxa de $60 \%$ destes casos.

A investigação das condições com que o trabalhador executa suas atividades é de elevada pertinência, tanto para o trabalhador, que é o principal afetado, quanto para a organização que é atingida de diversas maneiras, tendo no absenteísmo seu principal indicador. Neste contexto, Chechetto, Madeira e Longen (2016), argumentam que os riscos associados a lesões na coluna lombar decorrentes do trabalho devem ser objeto de estudo constante de pesquisas, o que implica em buscar melhorias práticas nas condições de trabalho.

No decorrer dos anos, diversas técnicas de avaliação dos riscos relacionados à movimentação manual de cargas foram desenvolvidas. Dentre estas, a mais difundida no Brasil, e também em países como Estados Unidos, Canadá, Reino Unido e Austrália é aquela conhecida por Equação de NIOSH, criada no âmbito da agência norte-americana National Institute for Occupational Safety and Health (NIOSH) no ano 1981, e que foi revisada resultando em sua versão mais recente, em 1994 (Waters, PutzAnderson e Garg, 1994). Teixeira (2004) explica que o objetivo da aplicação desta equação é prevenir e reduzir a ocorrência de lesões por sobrecarga e lombalgia entre os trabalhadores, há uma necessidade em mensurar as tarefas corriqueiras do cotidiano industrial de manipulação manual de cargas.

De um modo geral, a problemática de pesquisa rodeia os locais de trabalho que apresentam um grande número de tarefas envolvendo múltiplas alturas, frequências e ângulos de assimetria corporal, as quais deveriam ser mensuradas pela aplicação da Equação de NIOSH composta, desenvolvida especialmente para este fim e não pela Equaçãode NIOSH para tarefas simples. No Brasil 
o principal prejudicado é o trabalhador, que trabalha em postos de trabalho com riscos subdimensionados.

A Equação de NIOSH, em grande maioria dos trabalhos refere-se às tarefas denominadas simples (RIBEIRO et al.,2009; MORIRA et al.,2015; NAIDITCH et al.,2020) em contextos de tarefas compostas, o que não reflete a situação real comumente presente nas organizações. Assim temos uma lacuna no que diz respeito a trabalhos que explicitem e demonstram a aplicação da Equação de NIOSH para tarefas compostas

Desta maneira, apesar desta técnica ter sido formalmente publicada há mais de duas décadas, ainda há uma lacuna importante na literatura científica, a qual demonstre de forma mais didática e transparente seu uso. Contribuir para o preenchimento desta lacuna consiste uma das premissas deste trabalho.

Sendo assim, o presente trabalho tem o objetivo apresentar por meio da avaliação de uma situação cotidiana os encaminhamentos teóricos e práticos aplicáveis durante a utilização da avaliação de NIOSH para tarefas compostas, tendo como objeto de análise um posto de trabalho de maior complexidade contemplando a desmontagem de paletes de bebidas.

\section{Referencial teórico}

O referencial teórico, foi segmentado em três frentes, sendo a primeira com foco na contextualização da Ergonomia no Brasil e seu desenvolvimento, seguido da movimentação manual de cargas e, por fim, a Equação de NIOSH. Ao longo do texto estes conteúdos são apresentados e acabam dialogando para a construção de um marco teórico para o trabalho.

A Ergonomia em muitas situações, de maneira errônea, é atrelada como um campo de estudos que visa apenas a preservação da saúde e conforto do trabalhador, e deixa de lado o contexto organizacional das empresas. Silva, Lacombe, Tavares, Varela e Lellis (2010) discorrem que o desempenho produtivo de uma organização, e a produtividade dos trabalhadores é reflexo das condições ergonômicas que ela disponibiliza, proporcionando segurança, saúde e satisfação para seus trabalhadores. Em complemento, Moura et al. (2019) argumentam que as diretrizes ergonômicas alinhadas as técnicas de desenvolvimento de produtos, são eficientes para atender as necessidades específicas dos projetos de produtos.

Além disso, a participação dos trabalhadores é essencial para implementar e atualizar os meios de trabalho e consequentemente, reduzir o absenteísmo, aumentar produtividade, a qualidade de vida no trabalho e a qualidade do produto. Neste sentido, Wilson (2014) ressalta que talvez venha ser mais fácil reconhecer a ausência da Ergonomia nas organizações do que propriamente defini-la. 
Os estudos relacionados a Ergonomia tiveram seu início no Brasil em meados da década de 1960. Lucio, Alves, Razza, Silva e Paschoarelli (2010) ponderam que a Ergonomia no Brasil possui grande influência da escola francesa, um dos motivos seria o grande número de pesquisadores brasileiros que foram para França cursar mestrado e doutorado sob orientação dos professores Alain Wisner e Maurice Montmollion. A Ergonomia teve grande crescimento no país após criação da Associação Brasileira de Ergonomia, que passou a organizar congressos de forma a promover um debate crítico sobre a produção científica entre pesquisadores nacionais e internacionais. Atualmente, segundo demonstram Moura, Bemvenuti e Franz (2020), a inserção da produção brasileira em Ergonomia em âmbito global vem ganhando força nos últimos anos e, apesar de sua posição relativamente discreta comparada às principais potências econômicas atuais, o Brasil assume um destaque como protagonista da Ergonomia na América Latina.

Não obstante, um olhar pormenorizado com o foco em riscos ergonômicos mais específicos pode revelar aspectos importantes. A título exemplo, o manuseio manual de cargas consiste em um elemento que, de acordo com Kroemer e Grandjean (2005) é dos grandes responsáveis pelo absenteísmo e desgaste excessivo dos trabalhadores. Os principais distúrbios estão frequentemente vinculados à coluna vertebral. O absenteísmo por sua vez, consiste em um elemento particularmente crítico que pode realmente estar a associado a problemas envolvendo a falta de Ergonomia e carece para sua compreensão da gestão adequada de indicadores. Martins, Bacelar, Bonfin, Rodrigues e Xerez (2017) reportam que a atividade de carregamento de cargas manual depende do esforço físico do trabalhador, estando suscetíveis a lesões na musculatura e nas articulações, bem como propensos a acidentes e doenças ocupacionais.

Um dos elementos propulsores da disseminação da Ergonomia nas organizações brasileiras foi o alto índice de afastamentos de trabalhadores registrados, devido inicialmente às Lesões por Esforço Repetitivo (LER) e distúrbios osteomusculares relacionados ao trabalho (DORT). Segundo Neto, Bittencourt, Nasrala, Sousa e Roder (2015) as principais patologias relacionada a estas síndromes são dorsalgia, lesões do ombro, tenossinovites e sinovites.

Moreira e Nunes (2016) argumentam que a dor na região lombar é um dos principais motivos de afastamentos por longos períodos, aqueles acima de 15 dias, e é a segunda maior causa de afastamentos por períodos curtos. Com efeito, o manuseio manual de cargas é um dos grandes responsáveis pelas perdas financeiras relacionadas ao trabalhador, revelando-se através de lesões e doenças e do próprio absenteísmo.

Silva, Alexandre e Sousa (2014) ressaltam que a falta de condições adequadas de trabalho contribui para o aumento do risco de doenças e lesões ocupacionais, e esses fatores acabam acarretando em grandes perdas financeiras para as empresas. Estes autores apontam que há uma grande dificuldade em quantificar as perdas econômicas relacionadas a Ergonomia, ou falta dela. Um 
caminho seria mensurar o risco associado às tarefas de manuseio manual de cargas, de forma a evitar as perdas financeiras e organizacionais. Por sua vez, Garg (1995) já ressaltava nos anos 1990 que o grande intuito da Equação de NIOSH era mensurar a demanda física, psicofísica e fisiológica da tarefa do trabalhador com vistas a avaliar o risco por sobrecarga e também lombalgia.

É no contexto da manutenção de cargas que se aplica a utilização da Equação de NIOSH, a qual considera sete parâmetros para estabelecer um índice para a tarefa executada pelo trabalhador. Estes parâmetros que compõem a equação são multiplicados, sendo que quanto menor for o resultado final, melhor será o índice daquela tarefa. Esta Equação de NIOSH foi publicada pela primeira vez no ano de 1981, e levava em consideração três critérios principais, biomecânica, psicofísica e fisiologia do trabalho, e passou por uma grande revisão dez anos depois. Waters, Putz-Anderson, Garg e Fine (1993) ressaltam que a revisão possibilitou que a equação protegesse uma taxa ainda maior de trabalhadores. NIOSH (2014), também aponta que a equação estabelece um Limite de Peso Recomendado (LPR) para a tarefa de manipulação manual de cargas, sendo o valor inicial é fixado em $23 \mathrm{~kg}$, pois este seria o valor máximo da carga aceitável em condições ideais de trabalho, para 99\% dos homens e 75\% das mulheres. O valor de $L P R$ valor vai sendo reduzido à medida que os parâmetros são ponderados na equação. Na Equação 1, são devem ser inseridos os parâmetros, conforme indicados pela Figura 1:

Figura 1

Visualização dos Parâmetros a Serem Inseridos na Equação de NIOSH
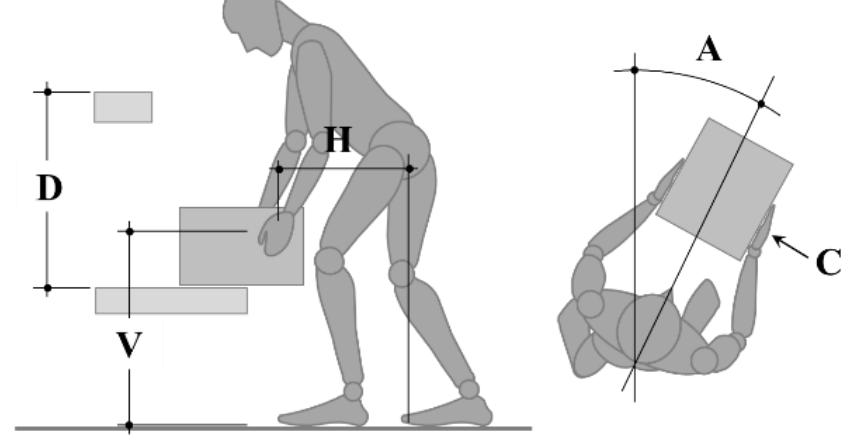

Fonte: Adaptado de Waters et al. (1994).

Equação $1 \mathrm{LPR}=23 \times(25 / H) \times[(1-(0,003 \times / \mathrm{V}-75 /)] \times[(0,82+(4,5 / D)] \times[1-(0,0032 \times A)] \times F \times C$

Onde:

$L P R \quad$ é o limite de peso recomendado a partir do efeito dos diversos fatores;

H é a distância da projeção horizontal entre o ponto médio entre os tornozelos do trabalhador e o centro da palma de sua mão apartir do momento em que preende a carga; 
$V \quad$ é a distância vertical do centro da palma da mão do operador ao ponto de apoio de seus pés no momento em que preende a carga;

$D \quad$ é a distância vertical percorrida pela carga entre seu ponto de origem e seu ponto de destino;

A é a soma dos ângulos acumulados pelo trabalho durante a manutenção da carga entre seu ponto de origem e seu ponto de destino;

C é o fator de pega, obtido tendo em conta as características de forma, preensão e altura de preensão do objeto (Ver Figura 3).

F $\quad$ é o fator de frequência obtido por meio de tabela padronizada (Ver Tabela 3).

A mesma equação é comumente representada indicando-se cada uma de suas partes como multiplicadores (ver Tabela 1). Neste ponto, um esclarecimento é válido, nenhum dos fatores pode ultrapassar o valor de 1 , tendo seus limites entre 0 e 1 . O intuito desta limitação no valor dos fatores da equação conforme pode-se conferir em Waters et al. (1994), é justamente para que em nenhuma tarefa o $L P R$ ultrapasse o valor final de $23 \mathrm{~kg}$ como peso máximo recomendado para aquela determinada tarefa.

\section{Tabela 1}

Representação da Equação 1 Por Meio de Multiplicadores

\begin{tabular}{c|c|c}
\hline Sigla & Nome do multiplicador & Parte da equação \\
\hline$M H$ & Multiplicador Horizontal & $\left(\frac{25}{H}\right)$ \\
\hline$M V$ & Multiplicador Vertical & {$[1-(0,003 x|\mathrm{v}-75|)]$} \\
\hline$M D$ & Multiplicador de Distância & {$[0,82+(4,5 / \mathrm{D})]$} \\
\hline$M A$ & Multiplicador de Assimetria & {$[1-(0,0032 \times \mathrm{A})]$} \\
\hline$M C$ & Multiplicador de Interface & Obtido pelo fluxo da Figura 3 \\
\hline$M F$ & Multiplicador de Frequência & Obtido pelo quadro da Tabela 3 \\
\hline
\end{tabular}

Fonte: Adaptado de Waters et al. (1994)

De acordo com Pereira, Debiase, Farias e Longen (2015), tendo em mãos o valor de LPR, é possível mensurar o risco da tarefa, denominado Índices de Levantamento Simples (ILS), o qual é obtido pela relação entre o valor de $L P R$ e valor da carga realmente manipulada. O valor de ILC é considerado limitado se for menor que 1, moderado se estiver entre 1 e 3 , e elevado se estiver acima de 3 sendo que, neste caso a tarefa caracteriza-se como inaceitável do ponto de vista ergonômico e deverá ser modificada imediatamente.

Fox, Lu, Occhipinti e Jaeger (2019) em estudo realizado sobre a utilização da Equação de NIOSH no decorrer dos anos, apontam que alguns importantes aspectos ergonômicos não são ponderados pela equação como por exemplo, idade e sexo, além de obesidade e histórico de dores nas costas dos trabalhadores. A mesma exposição a um risco (por exemplo, elevação manual) para pessoas diferentes 
não apresenta necessariamente o mesmo grau de risco. Os autores sugerem uma nova classificação do risco das tarefas de acordo com o Índice de Levantamento (IL) obtido, onde IL menor ou igual a 1 apresenta um risco muito baixo e $I L$ entre 1 e 1,5 apresenta um risco baixo, o risco seria moderado estaria entre 1,5 e 2 , alto entre 2 e 3 e muito alto quando o IL obtido fosse maior do que 3.

O valor de ILS é bastante útil e amplamente utilizado. Contudo, em situações contendo tarefas compostas por várias tarefas simples, não basta considerarmos a média dos ILS envolvidos nas tarefas. Conforme expõe Waters et al.(1994), ao realizar uma tarefa composta a fadiga do trabalhador não consiste em um somatório das fadigas sofridas por ele em cada nível e muito menos do valor médio de tais fadigas. Na verdade, no caso das tarefas compostas, uma simples média dos distintos índices simples deve dar lugar a uma compensação de efeitos que representa o risco real daquela tarefa em seu todo (BRASIL, 2002). Nestes casos, devem ser realizados procedimentos matemáticos específicos que permitam a obtenção do que se convencional chama de Índice de Levantamento Composto (ILC).

Waters et al. (1994) explica que o valor de ILC consiste no ILS de maior valor, acrescido dos ILS das demais tarefas simples, tendo em conta a sinergia dos efeitos acumulados das várias frequências inerentes a cada uma dessas tarefas. Portanto, a proposta de Waters et al. (1994) poderia ser representada pela operação matemática apresentada na Equação 2.

Equação $2 \quad I L C=I L S_{1}+\sum_{I=2}^{n} I L S_{i}$

Onde:

ILC é o índice de levantamento composto, considerando as diversas tarefas simples que compõe a atividade composta;

ILS 1 é o índice de levantamento da tarefa com o maior valor de ILS dentre as várias tarefas analisadas;

ILS $\quad$ é o índice de levantamento das várias tarefas simples, consideradas a partir da tarefa com o segundo maior ILS.

Para efeitos de melhor organização e compreensão dos procedimentos matemáticos utilizados para obtenção do ILC supõe-se que ILS sempre corresponderá ao ILS de maior valor. Os demais ILS, a partir do segundo maior valor, terão seus índices indicados sequencialmente, de sorte que tem-se uma apresentação dos dados conforme demonstrado abaixo:

$I L S_{1} \quad$ maior índice obtido

$I L S_{2} \quad$ segundo maior índice obtido

$I L S_{3} \quad$ terceiro maior índice obtido

$I L S_{n} \quad$ enésimo maior índice obtido

Também cabe lembrar aqui que o ILS de uma tarefa $i$ é obtido pela operação matemática apresentada na Equação 3. 
Equação $3 \quad I L S_{i}=\frac{Q_{i}}{L P R_{i}}$

Onde:

ILS $\quad$ é o Índice de Levantamento Simples de uma dada tarefa $i$, sob observação;

$Q_{i} \quad$ é a carga manuseada pelo operador na tafera sob observação;

$L P R_{i} \quad$ é o Limite de Peso Recomendado para a tarefa $i$, sob observação.

Neste ponto, e para efeitos de uma melhor compreensão da obtenção do ILC, é importante que se considere a fórmula apresentada na Equação 4.

Equação $4 \quad L P R_{i}=L P R I F_{i} \times F F_{(f i)}$

Onde:

$L P R I F_{i} \quad$ é o limite de peso recomendado idependente da frequência para a tarefa $i$, sob observação

$F F_{(f i)} \quad$ é o fator de frequência para a tarefa $i$, sob observação

Cabe destacar aqui que os valor dos fatores de frequência $F F$, assim como no caso dos ILS, também terão seus índices seguindo uma numeração sequencial, onde $F F_{(f 1)}$ corresponde à frequência identificada durante a obtenção do ILS de maior valor $\left(I L S_{1}\right)$, e assim suscetivamente. Didaticamente, estes índices são representados conforme segue:

$F F_{(f 1)} \quad$ é o fator de frequência obtido considerando-se a frequência de movimentação de carga realizada pelo operador na obtenção do do $I L C_{1}$

$F F_{(f 2)}$ é o fator de frequência obtido considerando-se a frequência de movimentação de carga realizada pelo operador na obtenção do do $I L C_{2}$

$F F_{(f 3)} \quad$ é o fator de frequência obtido considerando-se a frequência de movimentação de carga realizada pelo operador na obtenção do do $I L C_{3}$

$F F_{(f n)} \quad$ é o fator de frequência obtido considerando-se a frequência de movimentação de carga realizada pelo operador na obtenção do do $I L C_{n}$

Cabe recordar aqui que o valor de LPRIFi, é obtido pela Equação 5.

$$
\begin{array}{rl}
\text { Equação } 5 & L P R I F_{i}=23 \times\left(\frac{25}{H_{i}}\right) \times\left[(1-(0,003 \times|V i-75|)] \times\left(0,82+\left(\frac{4,5}{D_{i}}\right)\right) \times[1-\right. \\
& (0,0032 \times A)] \times C_{i}
\end{array}
$$

Substituindo-se a Equação 4 na Equação 3, obtem-se que:

Equação $6 \quad I L S_{i}=\frac{Q_{i}}{\operatorname{LPRIF}_{i} \times F F_{(f i)}}$ 
Considerando a substituição na Equação 2 pela Equação 6, obtém-se então a relação matemática apresentada na Equação 7.

Equação $7 \quad I L C=\frac{Q_{1}}{L P R I F_{1} \times F F_{(f 1)}}+\sum_{i=2}^{n} \Delta\left(\frac{Q_{i}}{L P R I F_{i} \times F F_{(j i)}}\right)$

Observe que na Equação 7 denota-se o símbolo $\Delta$, o qual indica que os somatórios a partir do segundo maior valor de ILS são efetuados considerando-se os acréscimos graduais da fadiga, que nesta equação são associados diretamente aos efeitos das frequências acumuladas. Dessa forma, poderia-se representar, sem prejuízo, a Equação 7 conforme se lê na Equação 8.

Equação 8

$$
I L C=\frac{Q_{1}}{L P R I F_{1} \times F F_{(f 1)}}+\sum_{i=2}^{n}\left(\frac{Q_{i}}{L P R I F_{i} \times \sum_{j=1}^{n} F F_{(f j)}}-\frac{Q_{i}}{L P R I F_{i} \times \sum_{j=1}^{n-1} F F_{(f j)}}\right)
$$

De onde se obtem que:

Equação 9

$$
I L C=\frac{Q_{1}}{L P R I F_{1} \times F F_{(f 1)}}+\sum_{I=2}^{n} \frac{Q_{i}}{L P R I F_{i}} \times\left(\frac{1}{\sum_{j=1}^{n} F F_{(f j)}}-\frac{1}{\sum_{j=1}^{n-1} F F_{(f j)}}\right)
$$

Uma vez compreendidos os procedimentos matemáticos apontados acima, suponha um caso hipotético em que tenhamos 4 tarefas, as quais irão compor os valores que culminarão na obtenção de um ILC. Neste caso teríamos que:

Equação 10

$$
I L S_{1}=\frac{Q_{1}}{L P R I F_{1} \times F F_{(f 1)}}
$$

consiste no maior ILS, dentre aqueles obtidos nas tarefas simples;

Equação 11

$$
I L S_{2}=\frac{Q_{2}}{L P R I F_{2} \times F F_{(f 2)}}
$$
é o segundo maior ILS, dentre os ILS obtidos nas tarefas simples;

Equação 12

$$
I L S_{3}=\frac{Q_{3}}{L P R I F_{3} \times F F_{(f 3)}}
$$

é o terceiro maior valor de ILS;

Equação 13

$$
I L S_{4}=\frac{Q_{4}}{L P R I F_{4} \times F F_{(f 4)}} \quad \text { é o quarto maior valor de ILS obtido. }
$$

Para poder utilizar a Equação 9 não utiliza-se simplesmente as frequências $F F_{(f 1)}, F F_{(f 2)}, F F_{(f 3)}$ e $F F_{(f 4)}$, nela apresentadas. Conforme sugere Waters et al. (1994) deve-se inserir os ILS na Equação 9, inserindo-se também o efeito cumulativo das frequências experimentadas pelo operador, conforme demonstrado na Equação 14.

$$
\begin{aligned}
& I L C=\frac{Q_{1}}{L P R I F_{1} \times F F_{(f 1)}}+\left[\frac{Q_{2}}{L P R I F_{2}} \times\left(\frac{1}{F F_{(f 1+f 2)}}-\frac{1}{F F_{(f 1)}}\right)\right]+\left[\frac{Q_{3}}{L P R I F_{3}} \times\left(\frac{1}{F F_{(f 1+f 2+f 3)}}-\right.\right. \\
& \left.\left.\frac{1}{F F_{(f 1+f 2)}}\right)\right]+\left[\frac{Q_{4}}{L P R I F_{4}} \times\left(\frac{1}{F F_{(f 1+f 2+f 3+f 4)}}-\frac{1}{F F_{(f 1+f 2+f 3)}}\right)\right]
\end{aligned}
$$


Uma vez compreendidos os procedimentos explanados acima, é possível compreender então sua aplicação em uma situação prática.

\section{Caminhos de pesquisa}

\subsection{Objeto de estudo}

O estudo caracteriza-se como um estudo de caso (YIN, 2015). O objeto de estudo no presente trabalho consistiu na aplicação da Equação de NIOSH para avaliação de tarefas compostas na rotina de um operador repositor de bebidas, o estudo caracte. Este tipo de atividade é comum no contexto de macromercados e supermercados existentes no sul Brasil, onde alguns funcionários trabalham levando paletes carregados de bebidas até os corredores e desmontam estes para reposição de fardos de bebidas em gôndolas.

É importante ressaltar que a análise ergonômica deste tipo de atividade não se restringe exclusivamente a aplicação de ferramentas como é o caso da Equação de NIOSH. Contudo, para fins do presente artigo, a consideração da atividade de desmontagem do palete e suas tarefas se mostra pertinente.

Com vistas à padronização dos conceitos tratados no presente artigo, cabe destacar que utilizase o termo tarefa para designar a atividade que é realizada pelo trabalhador. Esta é chamada de tarefa composta quando busca contemplar a operação da desmontagem de paletes como um todo, e é chamada simplesmente por tarefa, ou tarefa simples quando o objetivo é relatar a formação de apenas uma das camadas sob análise dos produtos nos paletes. Logo, a tarefa composta realizada durante a formação de paletes, é constituída por diversas tarefas simples voltadas à formação de níveis de camadas de paletes. Esta convenção é utilizada de forma a oferecer compatibilidade com os termos utilizados não só por Waters et al. (1993) e Waters et al. (1994), quanto o manual de aplicação da Norma Regulamentadora № 17 (NR-17) (BRASIL, 2002).

\subsection{Método e materiais}

As observações e análises utilizadas como amparo argumentativo neste artigo foram realizadas no contexto exclusivamente da atividade de desmontagem do palete e acondicionamento de bebidas em uma gôndola de supermercado. Inicialmente, foram realizados levantamento quanto às medidas e ritmo de trabalho tipicamente presentes na atividade objeto de estudo. Posteriormente, foram desenvolvidas as simulações, de acordo com os parâmetros estabelecidos pela Equação de NIOSH.

Os resultados obtidos durante a aplicação da avaliação são gradualmente discutidos e detalhados, de forma a atingir o objetivo deste artigo e devendo fornecer um caminho claro e aplicável para utilização em termos práticos do cálculo do índice composto para a equação NIOSH. A aplicação 
da Equação de NIOSH para tarefas compostas baseou-se nos seguintes passos, em harmonia com o que estabelece o Manual de aplicação da NR-17 (BRASIL,2002):

a) Coletou-se os dados e medições dos trabalhadores executando suas tarefas durante a jornada laboral;

b) Introduziu-se dos dados levantados na Equação de NIOSH, considerando cada montagem de um nível do palete como uma tarefa simples. Como resultado, obteve-se o ILS de cada tarefa simples;

c) A partir dos resultados obtidos no passo anterior (passo b), ordenou-se os valores obtidos de ILS de forma decrescente.

d) Efetuou-se o cálculo da agregação de riscos associados às diferentes tarefas simples (diferentes ILS).

\section{Resultados e discussão}

\subsection{Detalhamento do posto de trabalho}

A tarefa abrangida no presente artigo consiste no carregamento manual dos fardos com latas de cerveja, com peso médio de 5,90 kg do fardo, do palete até a gôndola. Esta tarefa enquadra-se em situações para avaliação utilizando a Equação de NIOSH composto por tratar-se de uma tarefa em que o trabalhador realiza posicionamento dos fardos através da movimentação manual da carga em um palete, perfazendo diversas camadas em diferentes alturas, conforme demonstrado na Figura 2.

\section{Figura 2}

Trabalhador Executando a Tarefa Avaliada

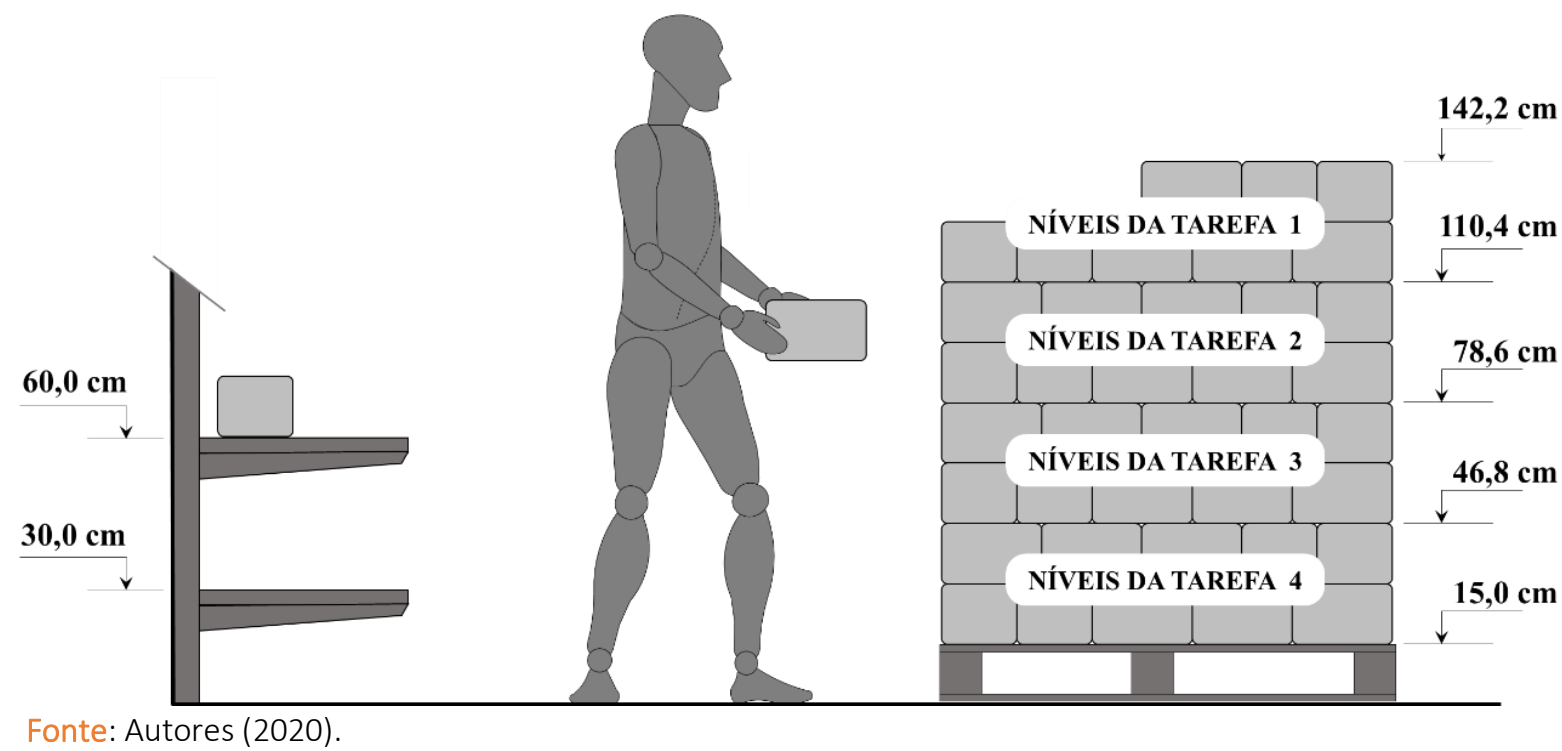


Para efeitos da presente análise, a atividade composta foi dividida em quatro fases, as quais serão chamadas tarefas. A primeira tarefa (Tarefa 1) ocorre quando o trabalhador pega o fardo, com peso de 5,90 kg provindos das camadas mais altas do palete $(110,4 \mathrm{~cm}$ de altura do piso) e os posiciona na praleteleira da gôndola (60 cm de altura). A segunda fase (Tarefa 2) consiste em pegar o fardo do produto na camada intermediária do palete, a uma altura de 78,6cm, logo abaixo da camada superior também colocando os fardos na prateleira a $60 \mathrm{~cm}$ de altura do piso. A terceira fase (Tarefa 3 ), por seu turno, consiste pegar o fardo de uma camada a $46,8 \mathrm{~cm}$ do piso e posicioná-lo na gôndola a $30 \mathrm{~cm}$ de altura. Por fim, na quarta fase (Tarefa 4) o operador pega os fardos que estão na base do palete, a $15 \mathrm{~cm}$ do piso, e os coloca na gôndola (30cm de altura), encerrando a operação de desmontagem do palete. A atividade em tela ocorre diariamente durante as primeiras 2 horas do turno de trabalho. Para utilizar a Equação de NIOSH, optou-se pelo levantamento dos fatores necessários para execução da equação de maneira separada.

Os valores mensurados durante levantamento no posto de trabalho são sumarizados na Tabela 2. A distância horizontal da carga varia ligeiramente das Tarefa 1 e 2, para as Tarefas 3 e 4 , devido ao fato do trabalhador pegar a carga próxima ao seu corpo e acabar esticando-se mais para pegar os fardos nos níveis mais baixos do palete.

\section{Tabela 2}

Valores Observados Durante a Observação do Posto de Trabalho

\begin{tabular}{c|c|c|c|c}
\hline & $\begin{array}{c}\text { Distância Horizontal } \\
\left(H_{i}\right)\end{array}$ & $\begin{array}{c}\text { Altura Inicial da carga } \\
\left(V_{i}\right)\end{array}$ & $\begin{array}{c}\text { Altura final da carga } \\
\left(V f_{i}\right)\end{array}$ & $\begin{array}{c}\text { Distância vertical } \\
\text { percorrida pela carga } \\
\left(V_{i}\right)\end{array}$ \\
\hline Tarefa 1 & $25 \mathrm{~cm}$ & $110,4 \mathrm{~cm}$ & $60 \mathrm{~cm}$ & $50,4 \mathrm{~cm}$ \\
\hline Tarefa 2 & $25 \mathrm{~cm}$ & $78,6 \mathrm{~cm}$ & $60 \mathrm{~cm}$ & $18,6 \mathrm{~cm}$ \\
\hline Tarefa 3 & $30 \mathrm{~cm}$ & $46,8 \mathrm{~cm}$ & $30 \mathrm{~cm}$ & $16,8 \mathrm{~cm}$ \\
\hline Tarefa 4 & $30 \mathrm{~cm}$ & $15,0 \mathrm{~cm}$ & $30 \mathrm{~cm}$ & $15,0 \mathrm{~cm}$ \\
\hline
\end{tabular}

Fonte: Autores (2020).

Em seguida, observaram-se os demais valores utilizados no cálculo do índice de NIOSH, sendo eles a Assimetria $\left(A_{i}\right)$, o Fator da Frequência da tarefa $\left(F F_{i}\right)$ e a Qualidade da Pega $\left(C_{i}\right)$. Para a observação do fator assimetria, os valores considerados foram os mesmos para todas as tarefas, gerando um ângulo de assimetria de 10 o entre os pontos de origem e de destino durante a movimentação da carga.

Para observação do fator de frequência $\left(F F_{i}\right)$ são necessárias algumas informações como a jornada diária dos trabalhadores, a mensuração de tempo de execução da tarefa e a frequência efetiva da tarefa. A frequência da manipulação da carga foi mensurada em 1 fardo por minuto para cada tarefa, em uma jornada de trabalho de 8 horas, onde os trabalhadores alocam aproximadamente 2 horas de sua jornada nesta operação, ficando ficando na classe sugerida pela ferramenta entre 2 horas e 8 horas. 
Garg e Kappelusch (2016) criticam esta faixa de jornada de trabalho, que penaliza da mesma forma uma tarefa executada por 8 horas quanto uma tarefa executada em 2 horas.

Após mensurar a frequência das tarefas executadas dos trabalhadores por minuto, o próximo consiste em obter o índice $F F_{i}$, tomando por base os valores sugeridos pelo manual de aplicação da NR17 (BRASIL, 2002), utilizando os valores apresentados na Tabela 3. Assim, o índice de frequência foi estimado em 0,75, o que equivale a uma frequência de 1 levantamentos por minuto em uma jornada de trabalho entre 2 e 8 horas.

Tabela 3

Índices de Frequência Para a Equação de NIOSH

\begin{tabular}{c|c|c|c|c|c|c}
\hline & \multicolumn{7}{|c}{ Duração do Trabalho } \\
\hline & \multicolumn{2}{|c|}{ Jornada $<1$} & \multicolumn{2}{c}{$1<=$ Jornada $<2$} & \multicolumn{2}{c}{$2<=$ Jornada $<=8$} \\
\hline levantamentos/min & $V<75$ & $V>75$ & $V<75$ & $V>75$ & $V<75$ & $V>75$ \\
\hline 0,2 & 1,00 & 1,00 & 0,95 & 0,95 & 0,85 & 0,85 \\
\hline 0,5 & 0,97 & 0,97 & 0,92 & 0,92 & 0,81 & 0,81 \\
\hline 1,0 & 0,94 & 0,94 & 0,88 & 0,88 & 0,75 & 0,75 \\
\hline 2,0 & 0,91 & 0,91 & 0,84 & 0,84 & 0,65 & 0,65 \\
\hline 3,0 & 0,88 & 0,88 & 0,79 & 0,79 & 0,55 & 0,55 \\
\hline 4,0 & 0,84 & 0,84 & 0,72 & 0,72 & 0,45 & 0,45 \\
\hline 5,0 & 0,80 & 0,80 & 0,60 & 0,60 & 0,35 & 0,35 \\
\hline 6,0 & 0,75 & 0,75 & 0,50 & 0,50 & 0,27 & 0,27 \\
\hline 7,0 & 0,70 & 0,70 & 0,42 & 0,42 & 0,22 & 0,22 \\
\hline 8,0 & 0,60 & 0,60 & 0,35 & 0,35 & 0,18 & 0,18 \\
\hline 9,0 & 0,52 & 0,52 & 0,30 & 0,30 & 0,00 & 0,15 \\
\hline 10,0 & 0,45 & 0,45 & 0,26 & 0,26 & 0,00 & 0,13 \\
\hline 11,0 & 0,41 & 0,41 & 0,00 & 0,23 & 0,00 & 0,00 \\
\hline 12,0 & 0,37 & 0,37 & 0,00 & 0,21 & 0,00 & 0,00 \\
\hline 13,0 & 0,00 & 0,34 & 0,00 & 0,00 & 0,00 & 0,00 \\
\hline 14,0 & 0,00 & 0,31 & 0,00 & 0,00 & 0,00 & 0,00 \\
\hline 15,0 & 0,00 & 0,28 & 0,00 & 0,00 & 0,00 & 0,00 \\
\hline 16,0 & 0,00 & 0,00 & 0,00 & 0,00 & 0,00 & 0,00 \\
\hline
\end{tabular}

Os valores de $V$ estão em $\mathrm{cm}$. Para frequências inferiores a 5 minutos, utilizar $\mathrm{F}=0,2$ elevações por minuto Fonte: BRASIL (2002).

Outro elemento que foi considerado foi a qualidade da pega $\left(C_{i}\right)$ da carga, também chamada de fator de interface, sendo que Waters et al. (1994) destacam que o simples fato da carga ter alças pode acarretar em uma redução de esforços entre as taxas de 7\% e 11\%. No caso do local sob análise a pega da carga foi classificada como má para todas as tarefas de acordo com as características apresentadas na Figura 3, pois o fardo do produto não possui apoios ou alças. 
Figura 3

Fluxograma para Tomada de Decisão Quanto ao Fator de Interface

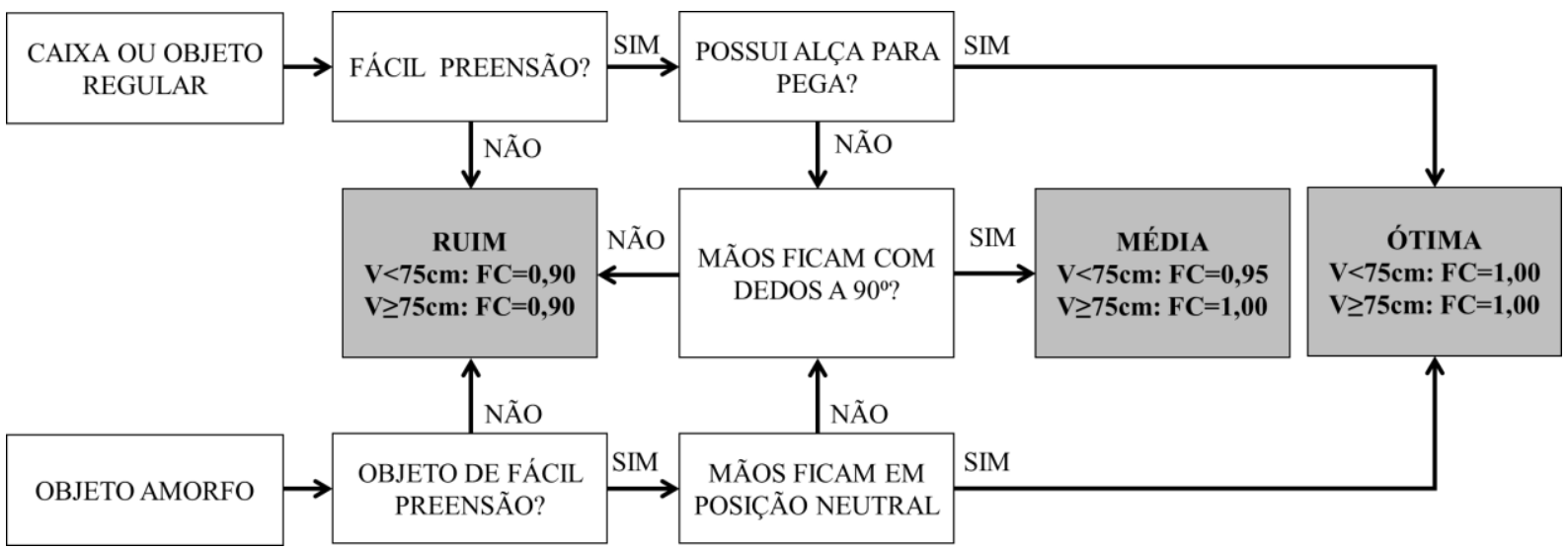

Fonte: Adaptado de Waters et al. (1994).

No caso das tarefas avaliadas, o valor a ser considerado e inserido na Equação de NIOSH foi 1,00 e 0,95, de acordo com a altura inicial da carga que o trabalhador movimenta.

\subsection{Resultados obtidos com aplicação dos valores analisados nas tarefas simples}

Os primeiros cálculos pelo NIOSH para tarefas compostas seguem os mesmos procedimentos da equação para tarefas simples. Os fatores a serem inseridos na equação estão dispostos na Tabela 4, de forma a facilitar a compreensão e a execução do cálculo.

\section{Tabela 4}

Dados Levantados Para as Tarefas Simples em Observação In Loco

\begin{tabular}{l|c|c|c|c}
\hline \multicolumn{1}{c|}{ Variável } & $\begin{array}{c}\text { Tarefa 1 } \\
i=1\end{array}$ & $\begin{array}{c}\text { Tarefa 2 } \\
i=2\end{array}$ & $\begin{array}{c}\text { Tarefa 3 } \\
i=3\end{array}$ & $\begin{array}{c}\text { Tarefa 4 } \\
i=4\end{array}$ \\
\hline$Q_{i}(\mathrm{~kg})$ & $5,90 \mathrm{~kg}$ & $5,90 \mathrm{~kg}$ & $5,90 \mathrm{~kg}$ & $5,90 \mathrm{~kg}$ \\
\hline$H_{i}(\mathrm{~cm})$ & $25 \mathrm{~cm}$ & $25 \mathrm{~cm}$ & $30 \mathrm{~cm}$ & $30 \mathrm{~cm}$ \\
\hline$V_{i}(\mathrm{~cm})$ & $110,4 \mathrm{~cm}$ & $78,6 \mathrm{~cm}$ & $46,8 \mathrm{~cm}$ & $15,0 \mathrm{~cm}$ \\
\hline$D_{i}(\mathrm{~cm})$ & $50,4 \mathrm{~cm}$ & $18,6 \mathrm{~cm}$ & $16,8 \mathrm{~cm}$ & $15,0 \mathrm{~cm}$ \\
\hline$A_{i}$ (graus) & $10 \circ$ & $10 \cong$ & $10 \cong$ & $10 \cong$ \\
\hline$C_{i}$ o & 1,00 (Média) & 1,00 (Média) & 0,95 (Ruim) & 0,95 (Ruim) \\
\hline$F_{i}$ (levantamentos/min) & 1 & 1 & 1 & 1 \\
\hline
\end{tabular}

Fonte: Autores (2020).

Os resultados obtidos com a aplicação dos valores apresentados na Tabela 4 podem ser dispostos por meio dos multiplicadores sugerido na Tabela 5. 
Tabela 5

Resultados Dispostos na Forma de Multiplicadores Para as Tarefas Analisadas

\begin{tabular}{c|c|c|c|c|c}
\hline \multicolumn{2}{c|}{ Partes da equação e variáveis resultantes } & $\begin{array}{c}\text { Tarefa 1 } \\
i=1\end{array}$ & $\begin{array}{c}\text { Tarefa 2 } \\
i=2\end{array}$ & $\begin{array}{c}\text { Tarefa 3 } \\
i=3\end{array}$ & $\begin{array}{c}\text { Tarefa 4 } \\
i=4\end{array}$ \\
\hline$M H_{i}$ & Multiplicador Horizontal & 1,000 & 1,000 & 0,833 & 0,833 \\
\hline$M V_{i}$ & Multiplicador Vertical & 0,894 & 0,989 & 0,915 & 0,820 \\
\hline$M D_{i}$ & Multiplicador de Distância & 0,909 & 1,000 & 1,000 & 1,000 \\
\hline$M A_{i}$ & Multiplicador de Assimetria & 0,968 & 0,968 & 0,968 & 0,968 \\
\hline$M C_{i}$ & Multiplicador de Interface & 1,000 & 1,000 & 0,950 & 0,950 \\
\hline$L P R I F_{i}$ & Limite de Peso Recomendado & 18,094 & 22,024 & 16,135 & 14,453 \\
\hline$M F_{i}$ & Independente da Frequência (kg) & & & & \\
\hline$L P R_{i}$ & Multiplicador de Frequência & 0,750 & 0,750 & 0,750 & 0,750 \\
\hline$I L S_{i}$ & Ínimite de Peso Recomendado de Levantamento Simples & 13,571 & 16,518 & 12,101 & 10,840 \\
\hline
\end{tabular}

Fonte: Autores (2020).

Dos dados considerados na operação em questão é possível notar diferenças entre as tarefas. A distância horizontal $\left(H_{i}\right)$ é um dos fatores responsáveis pela diferença, pois a tarefa de obter os fardos de produto nas camadas finais do palete exige que o trabalhador incline-se mais do que na execução das duas tarefas iniciais. Neste ponto observa-se também a aplicação da restrição sugerida por Waters et al. (1994), onde fator distância vertical $\left(V_{i}\right)$ da Tarefa 3, onde o mutiplicador de distância $M D_{3}$ e $M D_{4}$ tiveram seus valores limitados em 1,0. Cabe observar ainda que consta na Tabela 5 os valores obtidos para $L P R I F_{i}$ para cada uma das tarefas, apesar de não ganharem destaque no contexto do cálculo do ILS i, serão importantes durante o cálculo do ILC.

\subsection{Efetuando o cálculo da equação de NIOSH para tarefa composta}

A primeiro procedimento para obtenção do NIOSH composto consiste em colocar em ordem decrescente as três tarefas simples, tomando como referência seus ILS, conforme pode-se observar na Tabela 6.

\section{Tabela 6}

Tarefas Após Colocadas em Ordem Decrescente

\begin{tabular}{|c|c|c|c|}
\hline Ordem & Tarefa & $I L S_{i}$ & $L P R I F_{i}$ \\
\hline 10 & Tarefa 4 & $I L S_{4}=0,544$ & 14,453 \\
\hline 20 & Tarefa 3 & $I L S_{3}=0,488$ & 16,135 \\
\hline 30 & Tarefa 1 & $I L S_{2}=0,435$ & 18,094 \\
\hline 40 & Tarefa 2 & $I L S_{1}=0,357$ & 22,024 \\
\hline
\end{tabular}

Fonte: Autores (2020). 
O próximo passo consiste na aplicação da Equação 14, para que assim se obtenha o índice para tarefa composta, ou ILC. Um cuidado especial deve ser considerado durante a utilização do Fator Frequência $\left(F F_{i}\right)$ a ser inserido na referida equação. Para obtenção adequada do $F F_{i}$, deve-se primeiramente realizar a soma dos valores absolutos da frequência de levantamento da tarefa corrente e, mais os demais valores absolutos das frequências daquelas tarefas que já foram executadsd. Após a soma, atribui-se o índice da frequência na equação, conforme valor estipulado consultando a Tabela 3. Seguindo esta linha de racicínio, e observando-se a 0 , temos por exemplo, que o $I L S_{3}$ é o índice de levantamento da Tarefa 1 (3o lugar na ordenação), calculado com o acréscimo da frequência das tarefa anteriores (Tarefa 4 e Tarefa 3), ou seja, $F F_{(f 4+f 3+f 1)}=F F_{(1+1+1)}=F F_{(3)}$, sendo neste caso pela Tabela 3 igual a 0,550. A recomendação da Equação de NIOSH em considerar a soma das frequências das atividades foi concebida para determinar o efeito global para todas as tarefas que compõem a tarefa composta. Em sua primeira versão, a equação ponderava o risco estimando a média dos índices obtidos das tarefas simples (Teixeira, 2004), contudo, isso foi alterado a partir da proposta reformulada na revisão da equação.

Desde ponto em diante, a consideração do LPRIF torna-se particularmente importante. Como as frequências passam a influênciar significativamente os cálulos, o uso da equação conforme apresentado na Equação 14 mostra-se bastante útil. Sendo assim, a primeira parcela a ser calculada na Equação 14 é aquela consoante ao ILS $S_{1}$, ou seja, o ILS de maior valor. Neste caso tem-se o procedimento matemático apresentado na Equação 15, abaixo.

Equação $15 \quad I L S_{1}=\frac{Q_{1}}{L P R I F_{1} \times F F_{(f 1)}}=\frac{5,90}{14,453 \times 0,750}=0,544$

Note que o valores obtido na Equação 15 consiste exatamente o mesmo, já que aqui temos somente o $F F_{1}$, já considerado durante o cáculo do $I L S_{1}$.

A seguir é necessário proceder o cálculo das demais parcelas existentes na Equação 14 as quais consideram o efeito acumulativo dos esforços do trabalhador durante a atividade da montagem do palete. Para tanto, considere-se o procedimento matemático presente na Equação 16.

Equação $16 \quad \frac{Q_{2}}{\operatorname{LPRIF}_{2}} \times\left(\frac{1}{F F_{(f 1+f 2)}}-\frac{1}{F F_{(f 1)}}\right)$

Para preenchimento dos valores na Equação 16, deve-se considerar o $Q_{2}$ como sendo a carga manipula na tarefa que gerou o segundo maior valor de ILS, sendo neste caso 5,90 kg, presente na 0.0 valor do LPRIF também deverá ser aquele consernente ao segundo maior valor de ILS, e poderá ser obtido da Tabela 3. Os valores de $F F_{(f 1+f 2)}$ e $F F_{(f 1)}$ devem ser obtidos a partir dos seguintes procedimentos: 
- $F F_{(f 1)}$ será obtido pela Tabela 3 utilizando-se como parâmetro uma frequência de 1 levantamentos/minuto e uma jornada de $2 \mathrm{~h}$ a $8 \mathrm{~h}$;

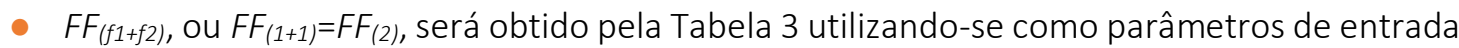
a soma das frequências $f 4$ (frequência da tarefa com maior ILS) e f3 (frequência da tafera com segundo maior ILS), tendo em conta as jornadas relativas às tarefas que associadas com essas frequências.

Dessa forma, obtem-se os valores apresentados na Equação 17.

Equação $17 \quad \frac{Q_{2}}{L P R I F_{2}} \times\left(\frac{1}{F F_{(f 1+f 2)}}-\frac{1}{F F_{(f 1)}}\right)=\frac{5,90}{16,135} \times\left(\frac{1}{0,650}-\frac{1}{0,750}\right)=0,075$

O próximo passo consistirá no cálculo da terceira parcela existente na Equação 14. Para tanto, o valor de Q continuará sendo aquele associado ao terceiro maior ILC, ou seja, 5,90 kg. O valor de LPRIF deverá ser extraído da Tabela 6 e também equivalerá aquele associado ao terceiro maior ILC. A frequências $F F(f 1+f 2+f 3)$ e $F F(f 1+f 2)$, serão obtidas através de procedimento similar aquele aplicado no caso da segunda parcela, detalhado anteriormente. Assim, tem-se que:

- $F F_{(f 1+f 2),} F F_{(1+1)}=F F_{(2)}$, será igual a 0,650 , segundo consulta à Tabela 3;

- $F F_{(f 1+f 2+f 3)}, F F_{(1+1+1)}=F F_{(3)}$, será igual a 0,550, conforme consulta à Tabela 3.

Tem-se, portanto, os procedimentos matemáticos apresentados na Equação 18.

Equação $18 \frac{Q_{3}}{\operatorname{LPRIF}_{3}} \times\left(\frac{1}{F F_{(f 1+f 2+f 3)}}-\frac{1}{F F_{(f 1+f 2)}}\right)=\frac{5,90}{18,094} \times\left(\frac{1}{0,550}-\frac{1}{0,650}\right)=0,091$

Por fim, o próximo passo consistirá no cálculo da quarta parcela existente na Equação 14. Continuamos tendo aqui o valor de $Q$ associado ao quarto maior ILC como sendo de 5,90 kg. O valor de LPRIF deverá ser extraído da Tabela 6 e também equivalerá aquele associado ao terceiro maior ILC. A frequências $F F(f 1+f 2+f 3+f 4)$ e $F F(f 1+f 2+f 3)$, serão obtidas através de procedimento similar as casos detalhados anteriormente. Assim, tem-se que:

- $F F_{(f 1+f 2+f 3)}, F F_{(1+1+1)}=F F_{(3)}$, será igual a 0,550 , segundo consulta à Tabela 3;

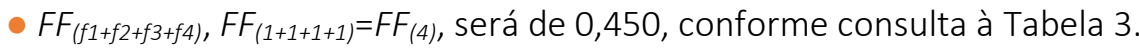

Tem-se, portanto, os procedimentos matemáticos apresentados na Equação 18.

Equação 19

$$
\frac{Q_{3}}{L P R I F_{3}} \times\left(\frac{1}{F F_{(f 1+f 2+f 3+f 4)}}-\frac{1}{F F_{(f 1+f 2+f 3)}}\right)=\frac{5,90}{20,024} \times\left(\frac{1}{0,450}-\frac{1}{0,550}\right)=0,119
$$

Se a tarefa composta sob análise contivesse cinco ou mais tarefas, os procedimentos apresentados acima seriam seguidos até o número de tarefas existentes, perfazendo assim cada uma das parcelas da equação que permite a obtenção do ILC, representada em sua forma genérica na Equação 9 e exemplificada para o caso de quatro tarefas na Equação 14. No caso particular da presente 
análise tem-se os procedimentos matemáticos a seguir, os quais buscam sumarizar os resultados indicados anteriormente pela substituição direta na Equação 14.

Equação 20

$$
\begin{aligned}
& I L C=\frac{5,90}{14,453 \times 0,750}+\left[\frac{5,90}{16,135} \times\left(\frac{1}{0,650}-\frac{1}{0,750}\right)\right]+\left[\frac{5,90}{18,094} \times\left(\frac{1}{0,550}-\frac{1}{0,650}\right)\right]+\left[\frac{5,90}{20,024} \times\right. \\
& \left.\left(\frac{1}{0,450}-\frac{1}{0,550}\right)\right]
\end{aligned}
$$

Equação $21 \quad I L C=0,544+0,075+0,091+0,119=0,829$

\subsection{Discussão dos resultados}

Conforme pode-se observar pelo resultado obtido após o procedimento matemático efetuado na Equação 21, o Índice de Levantamento Composto (ILC) para a tarefa composta abrangida no presente artigo é de 0,829. O valor obtido ainda denotaria que o trabalhador possui um risco baixo de vir a desenvolver alguma lesão, ou sofrer algum acidente, se a tarefa como um todo não sofrer alterações de melhorias. Nota-se pelos desdobramentos dos cálculos na abordagem apresentada neste trabalho que o uso dos valores do LPRIF (Limite de Peso Recomendado Independente da Frequência) pode simplificar significamente a obtençao de resultados.

Ao traçar uma comparação com os resultados inicialmente obtidos pela aplicação da equação NIOSH considerando somente as tarefas simples (Ver Tabela 6), percebe-se que houve um considerável aumento no índice obtido, ficando em torno do dobro do valor médio dos ILS individuais obtido inicialmente $\left(\overline{\operatorname{LI}_{l}}=0,456\right)$. Portanto, percebe-se que se os riscos da tarefa de desmontagem de palete fossem calculados à luz da Equação de NIOSH para tarefas simples, ou se fosse realizada apenas uma média aritmética entre os índices das tarefas simples, estes riscos seriam minimizados. Tal fato poderia acarretar em agravos à saúde dos trabalhadores que exercem essa tarefa.

No caso deste estudo, o ILC da tarefa composta representou um valor quase duas vezes maior do que se fosse realizada uma média das tarefas simples, o que reforça o fato de que o cálculo do ILC considera não uma média das fadigas e sim um acúmulo delas. Tendo em conta o exposto, fica evidente que o índice de NIOSH para tarefas compostas nos permite avaliar de forma mais criteriosa a tarefa como um todo.

\section{Conclusões}

Este trabalho apresentou por meio da avaliação de uma situação cotidiana os encaminhamentos teóricos e práticos aplicáveis durante a utilização da avaliação de NIOSH para tarefas compostas. O objeto de análise foi um posto de trabalho de maior complexidade contemplando a desmontagem de paletes de bebidas. 
A abordagem presente neste trabalho, embora não carregue um caráter inovador, contribui significativamente para a promoção do uso e aplicação precisa da Equação de NIOSH composta. Verificou-se que os cálculos na presente abordagem, onde se faz o uso dos valores do LPRIF simplifica bastante a obtenção de resultados, sem perda de acurácia.

O trabalho permitiu uma importante reflexão, onde analisar tarefas compostas de movimentação manual cargas, sob o prisma da Equação de NIOSH para tarefas simples pode acarretar em percepções equivocadas das situações de trabalho e consequentemente em danos à saúde do trabalhador. No caso deste estudo, o ILC da tarefa composta representou um valor quase duas vezes maior do que se fosse realizada uma média das tarefas simples, o que reforça o fato de que o cálculo do ILC considera não uma média das fadigas e sim um acúmulo delas.

Em adendo, o presente trabalho demonstrou que a utilização da Equação de NIOSH composta, desde que feita com parcimônia, pode trazer uma avaliação mais acurada e criteriosa dos riscos relativos à manutenção de cargas. Contudo, para isso é importante que o uso dos valores das frequências durante os procedimentos de cálculo seja feito de forma adequada, evitando-se eventuais distorções nos resultados.

\section{Referências}

Chechetto, S.T, Madeira, K., \& Longen, W.C. (2016). Identificação do risco lombar no trabalho de empacotamento manual de sacas de arroz. Revista Produção Online, v.16, n.2, p.395-407. https://doi.org/10.14488/1676-1901.v16i2.1780

BRASIL. (2007). Ministério do Trabalho e Emprego. Norma Regulamentadora №17 - Ergonomia. https://www.gov.br/trabalho-e-previdencia/pt-br/composição/orgaos-especi ficos/secretariade-trabalho/inspecao/seguranca-e-saude-no-trabalho/ctpp-nrs/norma-regulamentadora-no17-nr-17

BRASIL.. (2002). Ministério do Trabalho e Emprego. Manual de aplicação da Norma Regulamentadora no 17. Brasília: Ministério do Trabalho. Recuperado de http://www.ergonomia.ufpr.br/MANUAL_NR_17.pdf.

Fox, R.R., Lu, M.L., Occhipinti, E., \& Jaeger, M. (2019). Understanding outcome metrics of the revised NIOSH lifting equation. Applied ergonomics, v.81, p.1-6. https://doi.org/10.1016/j.apergo.2019.102897

Garg, A. (1995). Revised NIOSH equation for manual lifting: a method for job evaluation. AAOHN J, v.43, n.4, p.211-216. https://journals.sagepub.com/doi/pdf/10.1177/216507999504300408

Garg, A., \& Kapellusch, J.M. (2016). The cumulative lifting index (CULI) for the revised NIOSH lifting equation: quantifying risk for workers with job rotation. Human factors, v.58, n.5, p.683-694. https://doi.org/10.1177/0018720815627405

lida, I., \& Guimarães, L.B.M. (2005). Ergonomia: Projeto e Produção. 3 ed. São Paulo: Edgard Blücher. 
Kroemer, K. H. E., \& Grandjean, E. (2005). Manual de ergonomia: Adaptando o trabalho ao homem. Porto Alegre: Bookman. 328p.

Lucio, C.C., Alves, S.A., Razza, B.M., Silva, J.C.P., \& Paschoarelli, L.C. (2010). Trajetória da Ergonomia no Brasil: aspectos expressivos da aplicação em design. In: Silva, J.C.P., \& Paschoarelli, L.C. A evolução histórica da ergonomia no mundo e seus pioneiros. São Paulo: Editora Unesp, cap. 10, p.91-103. https://books.scielo.org/id/b5b72/pdf/silva-9788579831201-11.pdf?

Martins, J.R., Bacelar, T.C., Bonfim, W.B., Rodrigues, M.V., \& Xerez, F.C. (2017). Análise ergonômica no transporte manual de cargas: Um estudo de caso em uma empresa de produção de cimento. Revista GEPROS, v.12, n.1, p.269. https://doi.org/10.15675/gepros.v12i1.1627

Moreira, E. S, Chaves, C. A., dos Santos, J. C. D., \& Rodrigues, J. W. (2015). Melhorias Ergonômicas Utilizando a Equação Revisada de Levantamento Niosh. Revista de Ciências Exatas e Tecnologia, 10(10). https://doi.org/10.17921/1890-1793.2015v10n10p\%25p

Moreira, E.S., \& Nunes, L.E.N.P. (2016). A influência da ergonomia em melhorias produtivas utilizando a equação niosh. Revista Gestão Industrial, v.12, n.4. https://doi.org/10.3895/gi.v12n4.4537

Moura, C.R, Círico, E., Lafin, N.N.,Borges, T.L., \& da Silva, S. S. (2019). Desenvolvimento do conceito de uma plataforma de acesso a piscinas para pessoas com mobilidade reduzida. Exacta, 17(1). https://doi.org/10.5585/exactaep.v17n1.8049

Moura, H.M., Bemvenuti, R.H., \& Franz, L.A.S. (2020). Produção brasileira em Ergonomia no cenário internacional. Revista Prâksis - Dossiê: Trabalho, Saúde e Inclusão Social, a.17, v.1, p.31-56, jan./abr. https://doi.org/10.25112/rpr.v1i0.2042

Naiditch, I. F., Funck, M., Sander, N. E., \& Lerman, L. V. (2020). ESTUDO COMPARATIVO DOS IMPACTOS ERGONÔMICOS DA UTILIZAÇÃO DE PROCESSOS FÍSICOS E ELETRÔNICOS NO PODER JUDICIÁRIO. Produto \& Produção, 21(1). https://doi.org/10.22456/1983-8026.97785

Neto, E.N.; Bittencourt, W.S.; Nasrala, M.L.S; Sousa, F.P.; Roder, I.B. (2015).A influência do nexo técnico epidemiológico previdenciário sobre as notificações de LER/DORT no INSS. Journal of Health Sciences, v.16, n.3. https://doi.org/10.17921/2447-8938.2014v16n3p\%25p

NIOSH.(2014). National Institute for Occupational Safety Health. Local Health Departments and the NIOSH Health Hazard Evaluation Program: Working Together., DHHS (NIOSH) Publication No. 2014-113. https://doi.org/10.17921/2447-8938.2014v16n3p\%25p

Pereira, C.C., Debiase, D.F, Farias, J.M., \& Longen, W.C. (2015). Análise do Risco Ergonômico Lombar de Trabalhadores da Construção Civil Através do Método NIOSH. Produção Online, v.15, n.3. https://doi.org/10.14488/1676-1901.v15i3.1888

Ribeiro, I. A. V., Tereso, M. J. A., \& Abrahão, R. F. (2009). Ergonomic work analyses method in fresh tomato packing houses: manual material handling. Ciência Rural, 39(4), 1073-1079. https://doi.org/10.1590/S0103-84782009005000037

Silva, J.S., Lacombe, J.A., Tavares, E.M., Varela, A.M., \& Lellis, J.A. (2010). A ergonomia como um fator determinante no bom andamento da produção: um estudo de caso. Anagrama, v.4, n.1, p.114. https://doi.org/10.11606/issn.1982-1689.anagrama.2010.35485 
Silva, J.S., Alexandre, S.M.B., \& Sousa, M.N.A. (2014). Acidentes de trabalho e subnotificações: estudo com enfermeiros atuantes na atenção terciária. Ciência \& Desenvolvimento-Revista Eletrônica da FAINOR v.7, n.2. Recuperado de https://essentia.uvanet.br/index.php/ESSENTIA/article/view/75/84

Teixeira, E.R. (2004). Sistematização de procedimentos necessários à aplicação da ELN: Estudo descritivo da relação entre o IL da equação revisada do NIOSH e a incidência de lombalgia numa amostra de trabalhadores. 239 f. 2004. Dissertação. Mestrado em Engenharia Mecânica do Departamento de Engenharia Mecânica, Setor Tecnológico, Universidade Federal do Paraná, Curitiba. Recuperado de https://acervodigital.ufpr.br/bitstream/handle/1884/28901/D\%20\%20ELIANA\%20REMOR\%20 TEIXEIRA.pdf?sequence=1

Waters, T.R., Putz-Anderson, V., Garg, A., \& Fine, L.J. (1993). "Revised NIOSH equation for the design and evaluation of manual lifting tasks." Ergonomics, v.36, n.7, p. 749-776. https://doi.org/10.1080/00140139308967940

Waters, T.R., Putz-Anderson, V., \& Garg, A. (1994). Applications manual for the revised NIOSH lifting equation. National Institute for Occupational Health, Cincinnati, Ohio. https://doi.org/10.26616/NIOSHPUB94110revised092021external icon.

Waters, T.R., Lu, M.L., Piacitelli, L.A., Werren, D., \& Deddens, J.A. (2011) Efficacy of the revised NIOSH lifting equation to predict risk of low back pain due to manual lifting: expanded cross-sectional analysis. Journal of Occupational and Environmental Medicine, v.53, n.9, p.1061-1067. https://doi.org/10.1177/0018720813513608

Wilson, J.R. (2014) Fundamentals of systems ergonomics/human factors. Applied Ergonomics, v.45, n.1, p.5-13. http://dx.doi.org/10.1016/j.apergo.2013.03.021

Yin, R. K. (2015). Estudo de Caso-: Planejamento e métodos. Bookman editora. 